\title{
Is the Glasgow Prognostic Score Applicable to Both Early- and Advanced-Stage Gastric Cancers?
}

\author{
Tomoyuki Wakahara ${ }^{\mathrm{a}, \mathrm{b}}$, Nozomi Ueno ${ }^{\mathrm{a}}$, Tetsuo Maeda ${ }^{\mathrm{a}}$, Kiyonori Kanemitsu ${ }^{\mathrm{a}}$, \\ Takuro Yoshikawa ${ }^{\text {a }}$, Shinobu Tsuchida ${ }^{\text {a }}$, Akihiro Toyokawa ${ }^{\mathrm{a}}$
}

\begin{abstract}
Background: The Glasgow prognostic score (GPS) has been reported as a sensitive prognostic marker for gastric cancer. This study aimed to investigate whether the GPS is equally applicable to patients with early-stage and advanced-stage gastric cancers.

Methods: Patients $(n=544)$ who underwent elective gastrectomy for gastric cancer between 2007 and 2015 were retrospectively studied. GPSs of 2, 1, and 0 were allocated to patients with both an elevated Creactive protein level $(>1.0 \mathrm{mg} / \mathrm{dL})$ and hypoalbuminemia $(<3.5 \mathrm{mg} /$ $\mathrm{dL}$ ), patients with only one of these abnormalities, and patients with neither abnormality, respectively. The prognostic factors relevant to patients with early-stage (pStage I, $\mathrm{n}=304$ ) and advanced-stage (pStage II, III, and IV, n = 240) gastric cancer were analyzed through univariate and multivariate analyses.
\end{abstract}

Results: In the early-stage group, only the serum carbohydrate antigen (CA) 19-9 level $(\mathrm{P}=0.037)$ was a significant prognostic factor in the multivariate analysis; the GPS was not significant $(P=0.095)$. In the advanced-stage group, an American Society of Anesthesiologists physical status of 3 or $4(\mathrm{P}=0.032)$, elevated carcinoembryonic antigen (CEA) $(\mathrm{P}=0.043)$ and CA19-9 $(\mathrm{P}=0.045)$ levels, a GPS 1 - 2 (P $=0.017)$, and type 4 tumor $(\mathrm{P}=0.020)$ correlated significantly with worse overall survival.

Conclusions: GPS is a simple and useful prognostic score for patients with advanced-stage, but is not applicable to early-stage patients.

Keywords: Gastric cancer; Prognostic score; Survival

\section{Introduction}

In Japan, gastric cancer is a common type of malignancy. Here,

Manuscript submitted December 8, 2017, accepted December 27, 2017

aDepartment of Surgery, Yodogawa Christian Hospital, Osaka, Japan ${ }^{b}$ Corresponding Author: Tomoyuki Wakahara, Department of Surgery, Yodogawa Christian Hospital, 1-7-50, Kunijima, Higashi Yodogawa Ku, Osaka 5330024, Japan. Email: wakkan@mail.goo.ne.jp

doi: https://doi.org/10.14740/gr943w more than 132,000 patients were newly diagnosed with gastric cancer in 2011, and this disease was the third-leading cause of cancer-related deaths in 2014, after lung cancer and colorectal cancer [1]. To date, surgery has been a mainstay of treatment for gastric cancer; however, this treatment modality is associated with a relatively high morbidity rate $[2,3]$, and the longterm postoperative outcomes, especially of advanced cases, require improvement [4].

The selection of appropriate treatment for gastric cancer requires the identification of prognostic factors. Hypoalbuminemia has been reported to correlate with poor long-term outcomes in patients with various malignancies, including gastric cancer $[5,6]$, and an elevated serum C-reactive protein (CRP) level has been shown to predict poor prognosis [7]. The Glasgow prognostic score (GPS), which combines these two prognosticators, has been described as a more sensitive predictive marker of survival than either factor alone $[8,9]$. Although the GPS has been reported as a sensitive prognostic marker in gastric cancers [10-20], most previous studies included only advanced gastric cancer [18-20] or a mixture of various stages [12-17]; accordingly, the applicability of the GPS in patients with early-stage gastric cancer remains unknown. The present study aimed to investigate whether the GPS is equally applicable to patients with early-stage and advanced-stage gastric cancers.

\section{Materials and Methods}

A total of 565 consecutive patients with primary gastric cancer underwent elective surgery at the Yodogawa Christian Hospital between January 2007 and December 2015. Twenty-one patients were excluded from this study, including five who underwent pancreatoduodenectomy because of duodenal invasion, comorbid pancreatic cancer, or cholangial cancer; 12 who underwent gastrectomy for remnant stomach cancer; and four who underwent palliative partial gastrectomy without lymph node dissection. The clinicopathological information and long-term postoperative outcomes of the remaining 544 patients who underwent gastrectomy were retrospectively studied. In brief, D2 lymphadenectomy was performed in patients with T2 or deeper gastric cancer, or T1 gastric cancer with suspected lymph node metastasis, and D1 or D1+ lymphadenectomy was performed in patients with $\mathrm{T} 1$ gastric cancer, according to the Japanese gastric cancer treatment guideline (ver. 4) [21]. 
Table 1. Associations of Clinicopathological Characteristics With the Pathological Stage Among Gastric Cancer Cases

\begin{tabular}{|c|c|c|c|c|}
\hline & Total, $n=544$ & pStage $I, n=304$ & pStage II, III, IV, n = 240 & P value \\
\hline$\geq 75$ & 171 & $79(26.0 \%)$ & $92(38.3 \%)$ & \\
\hline Male & 379 & $206(67.8 \%)$ & $173(72.1 \%)$ & \\
\hline Female & 165 & $98(32.2 \%)$ & $67(27.9 \%)$ & \\
\hline$\geq 18$ & 496 & $284(93.4 \%)$ & $212(88.3 \%)$ & \\
\hline Comorbidity & & & & 0.931 \\
\hline$(+)$ & 366 & $205(67.4 \%)$ & $161(67.1 \%)$ & \\
\hline$(-)$ & 178 & $99(32.6 \%)$ & $79(32.9 \%)$ & \\
\hline ASA PS & & & & 0.116 \\
\hline eGFR $\left(\mathrm{mL} / \mathrm{min} / 1.73 \mathrm{~m}^{2}\right)$ & & & & 0.2132 \\
\hline$<60$ & 118 & $60(19.7 \%)$ & $58(24.2 \%)$ & \\
\hline$\geq 60$ & 426 & $244(80.3 \%)$ & $182(75.8 \%)$ & \\
\hline $\operatorname{Albumin}(\mathrm{g} / \mathrm{dL})^{\mathrm{a}}$ & & & & $<0.001^{*}$ \\
\hline$<3.5$ & 92 & $26(8.6 \%)$ & $66(27.5 \%)$ & \\
\hline$\geq 3.5$ & 434 & $267(87.8 \%)$ & $167(69.6 \%)$ & \\
\hline Lymphocytes (cells/ $\mu \mathrm{L})$ & & & & $0.018^{*}$ \\
\hline$<1,200$ & 111 & $51(16.8 \%)$ & $60(25.0 \%)$ & \\
\hline$\geq 1,200$ & 433 & $253(83.2 \%)$ & $180(75.0 \%)$ & \\
\hline $\mathrm{CRP}(\mathrm{mg} / \mathrm{dL})^{\mathrm{a}}$ & & & & $0.012 *$ \\
\hline$\leq 1.0$ & 496 & $284(93.4 \%)$ & $212(88.3 \%)$ & \\
\hline$>37$ & 70 & $21(6.9 \%)$ & $49(20.4 \%)$ & \\
\hline GPS $^{\mathrm{a}}$ & & & & $<0.001^{*}$ \\
\hline 0 & 407 & $252(82.9 \%)$ & $155(64.6 \%)$ & \\
\hline 1 & 97 & $35(11.5 \%)$ & $62(25.8 \%)$ & \\
\hline 2 & 20 & $4(1.3 \%)$ & $16(6.7 \%)$ & \\
\hline Macroscopic type & & & & $<0.001 *$ \\
\hline Type 4 & 28 & $1(0.3 \%)$ & $27(11.3 \%)$ & \\
\hline Other than type 4 & 516 & $303(99.7 \%)$ & $213(88.8 \%)$ & \\
\hline Tumor location & & & & $<0.001 *$ \\
\hline Upper third & 169 & $69(22.7 \%)$ & $100(41.7 \%)$ & \\
\hline Middle or lower third & 375 & $235(77.3 \%)$ & $140(58.3 \%)$ & \\
\hline Postoperative morbidity ${ }^{\mathrm{b}}$ & & & & $<0.001 *$ \\
\hline$(+)$ & 105 & $40(13.2 \%)$ & $65(27.1 \%)$ & \\
\hline$(-)$ & 439 & $264(86.8 \%)$ & $175(72.9 \%)$ & \\
\hline
\end{tabular}

BMI: body mass index; ASA PS: American Society of Anesthesiologists physical status; eGFR: estimated glomerular filtration rate; CRP: C-reactive

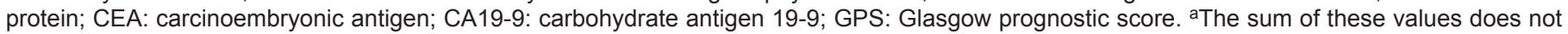
reach 544 because data were missing for some patients. ${ }^{b}$ Patients with grade II or higher postoperative complications (Clavien-Dindo classification) are classified as $(+)$. 
Table 2. Univariate and Multivariate Analyses of Clinicopathological Variables Associated With Overall Survival in All Gastric Cancer Patients

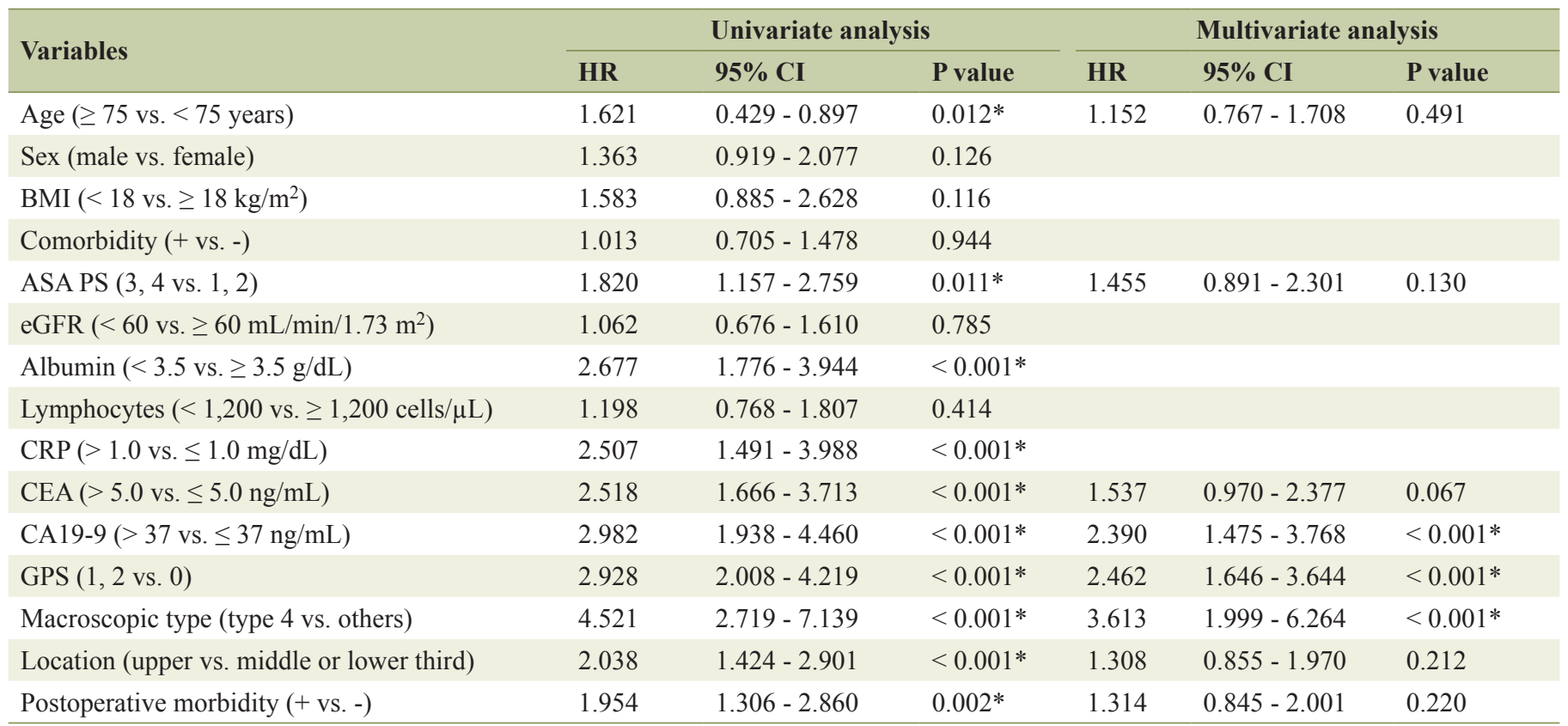

HR: hazard ratio; Cl: confidence interval; BMI: body mass index; ASA PS: American Society of Anesthesiologists physical status; eGFR: estimated glomerular filtration rate; CRP: C-reactive protein; CEA: carcinoembryonic antigen; CA19-9: carbohydrate antigen 19-9; GPS: Glasgow prognostic score.

GPSs were calculated using preoperative CRP and albumin levels; patients with both an elevated CRP level $(>1.0$ $\mathrm{mg} / \mathrm{dL})$ and hypoalbuminemia $(<3.5 \mathrm{mg} / \mathrm{dL})$, those with either abnormality, and patients with neither abnormality were allocated GPSs of 2, 1, and 0, respectively. Other laboratory data, including the estimated glomerular filtration rate (eGFR), lymphocyte count, and tumor markers, were also acquired preoperatively.

Pathological results were assessed according to the Japanese Classification of Gastric Carcinoma, third English edition [22]. Stage I gastric cancers include tumors confined to the mucosa or submucosa with 0 - 2 regional lymph node metastasis and tumors invading the muscularis propria without regional lymph node metastasis. Type 4 tumor means a diffuse, infiltrative tumor without marked ulceration or raised margins in which the gastric wall is thickened and indurated.

It was occasionally difficult to classify the tumor location as upper, middle, or lower third of the stomach as the tumors were often located along the borders between the upper, middle, and/or lower thirds or involved several areas. Thus, in this study, tumor locations were classified as "tumors including upper third" of the stomach, which required total gastrectomy or proximal gastrectomy, or "localized in the middle or lower third" of the stomach, which could be resected via distal gastrectomy or pylorus-preserving gastrectomy.

The protocol was approved by the institutional ethical committee on human experimentation, and was in accordance with the Helsinki Declaration of 1964 and later versions. Informed consent or a substitute for it was obtained from all pa- tients for being included in the study.

\section{Statistical analyses}

Intergroup comparisons of proportions or frequencies were performed using Fisher's exact test or the Chi-squared test. Survival curves were generated using the Kaplan-Meier method, and survival analyses and hazard ratio calculations were performed using a Cox proportional hazard model. Valuables with P values $<0.1$ in the univariate analysis were included in the multivariate analysis. All statistical analyses were performed using JMP software 12.2.0 (SAS Institute Inc., Cary, NC, USA).

\section{Results}

Table 1 lists the clinicopathological characteristics of the 544 patients with primary gastric cancer who underwent elective gastrectomy, as well as the associations of clinicopathological characteristics with pathological stage. The median follow-up period was 38.6 months (range: 0.2 - 99.8 months). Patients with advanced-stage gastric cancer (pStage II, III, and IV, $\mathrm{n}=240$ ) were significantly older than those with early-stage gastric cancer (pStage I, $\mathrm{n}=304)(\mathrm{P}<0.001)$. The proportions of patients with hypoalbuminemia $(\mathrm{P}<0.001)$, lower lymphocyte counts $(\mathrm{P}$ $=0.018)$, elevated serum CRP levels $(\mathrm{P}=0.012)$, and elevated serum tumor marker levels $(\mathrm{P}<0.001)$ were significantly higher among the advanced-stage group, which consequently had a significantly higher GPS (P $<0.001)$. Type 4 tumors were more 
Table 3. Univariate and Multivariate Analyses of Clinicopathological Variables Associated With Overall Survival in Patients With Stage I Gastric Cancer

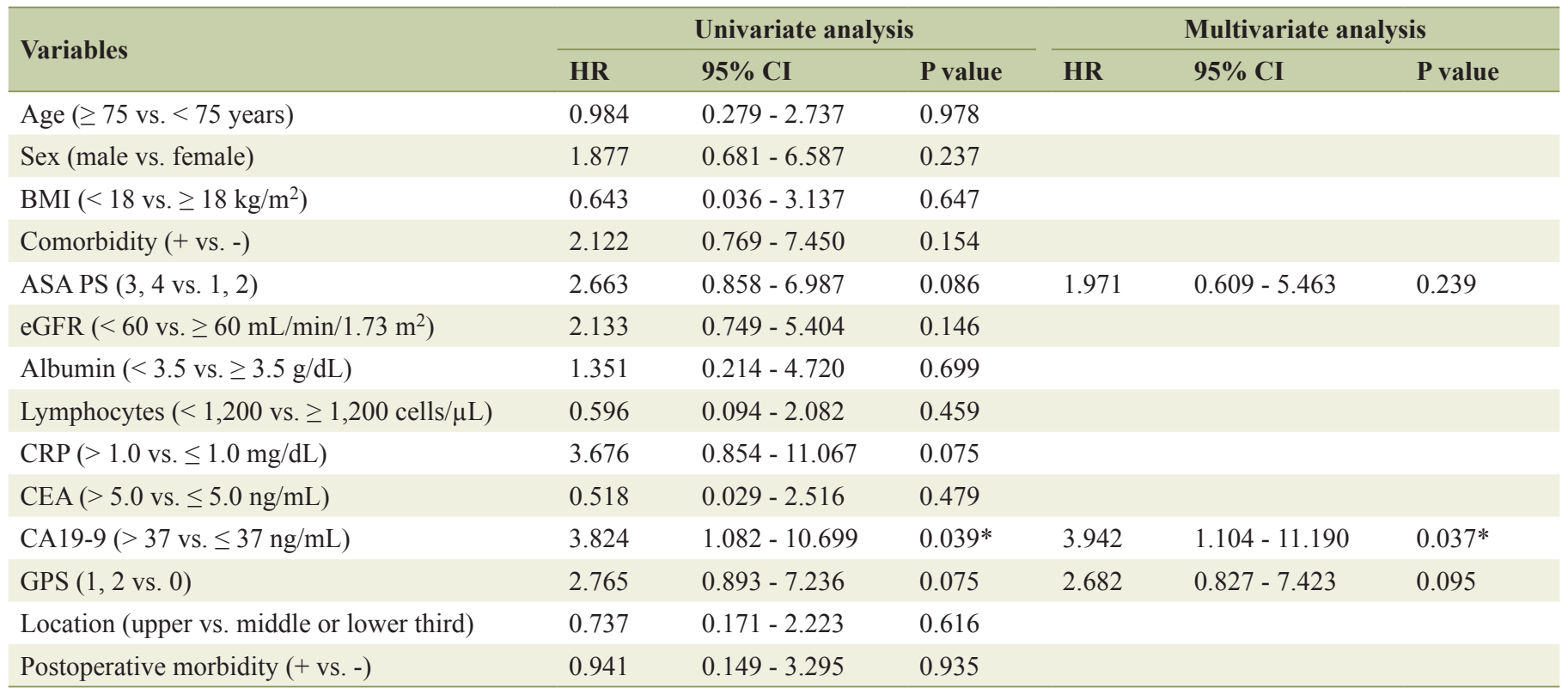

HR: hazard ratio; CI: confidence interval; BMI: body mass index; ASA PS: American Society of Anesthesiologists physical status; eGFR: estimated glomerular filtration rate; CRP: C-reactive protein; CEA: carcinoembryonic antigen; CA19-9: carbohydrate antigen 19-9; GPS: Glasgow prognostic score.

frequently detected in the advanced-stage group $(\mathrm{P}<0.001)$, and tumors in this group more frequently included the upper third of the stomach $(\mathrm{P}<0.001)$. The proportion of patients with grade II or higher postoperative morbidity was also significantly higher in the advanced-stage group $(\mathrm{P}<0.001)$.

The univariate and multivariate analyses of overall survival in the entire cohort of gastric cancer patients are shown in Table 2. An age of $\geq 75$ years $(P=0.012)$; an American Society of Anesthesiologists physical status (ASA PS) of 3 or 4 $(\mathrm{P}=0.011)$; hypoalbuminemia $(\mathrm{P}<0.001)$; elevated $\mathrm{CRP}(\mathrm{P}<$ $0.001)$, carcinoembryonic antigen $(\mathrm{CEA})(\mathrm{P}<0.001)$, and carbohydrate antigen (CA) 19-9 (P $<0.001)$ levels; a GPS of 1 or 2 $(\mathrm{P}<0.001)$; type 4 gastric cancer $(\mathrm{P}<0.001)$; tumors involving the upper third of the stomach $(\mathrm{P}<0.001)$; and postoperative morbidity $(\mathrm{P}=0.002)$ were identified as factors associated with reduced overall survival. In a multivariate analysis, an elevated CA19-9 level, GPS of 1 or 2, and type 4 tumor were significantly associated with worse overall survival (all $\mathrm{P}<0.001$ ).

In the early-stage group, only an elevated CA19-9 level was identified as a prognostic factor associated with worse overall survival in both the univariate $(\mathrm{P}=0.039)$ and multivariate analyses $(\mathrm{P}=0.037)$ (Table 3$)$. GPS was not a significant prognostic factor in this group $(\mathrm{P}=0.095)$ (Fig. 1).

In contrast, the significant prognostic factors identified in the advanced-stage group were similar to those identified for all gastric cancer cases (Table 4). In the univariate analysis, an ASA PS of 3 or $4(\mathrm{P}=0.033)$, hypoalbuminemia $(\mathrm{P}$ $=0.017)$, elevated CEA $(\mathrm{P}<0.001)$ and CA19-9 $(\mathrm{P}=0.006)$ levels, a GPS of 1 or $2(\mathrm{P}=0.002)$, type 4 gastric cancer $(\mathrm{P}=$ $0.007)$, and tumors involving the upper third of the stomach $(\mathrm{P}=0.025)$ were identified as factors associated with reduced overall survival. In a multivariate analysis, an ASA PS of 3 or $4(\mathrm{P}=0.032)$, elevated CEA $(\mathrm{P}=0.043)$ and CA19-9 $(\mathrm{P}=$ $0.045)$ levels, a GPS of 1 or $2(\mathrm{P}=0.017)$ (Fig. 2), and a type 4 tumor $(\mathrm{P}=0.020)$ were significantly associated with worse overall survival.

\section{Discussion}

GPS, which combines the CRP and albumin levels, was origi-

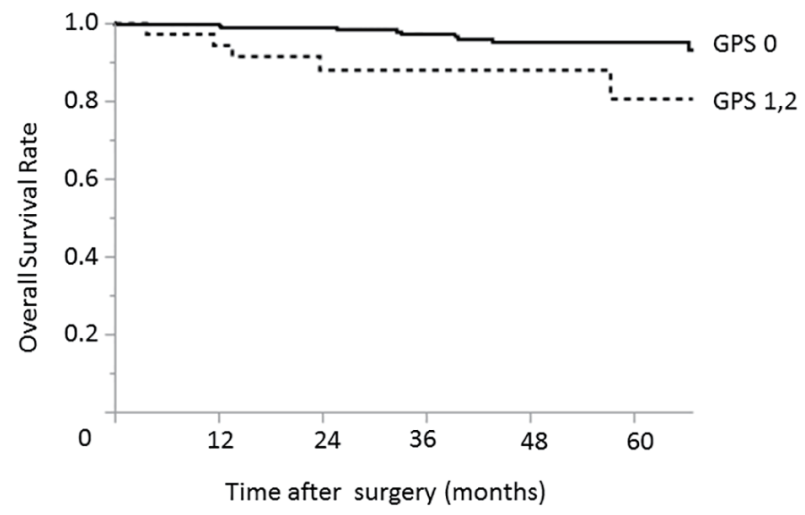

Figure 1. Relationship between the GPS and overall survival among patients with early-stage (pStage I) gastric cancer. The 3- and 5-year overall survival rates among patients with GPS of 0 were $97.1 \%$ and $95.1 \%$, respectively; the corresponding rates among patients with GPSs of $1-2$ were $88.1 \%$ and $80.7 \%$, respectively. GPS: Glasgow prognostic score. 
Table 4. Univariate and Multivariate Analyses of Clinicopathological Variables Associated With Overall Survival Among Patients With Stage II, III and IV Gastric Cancer

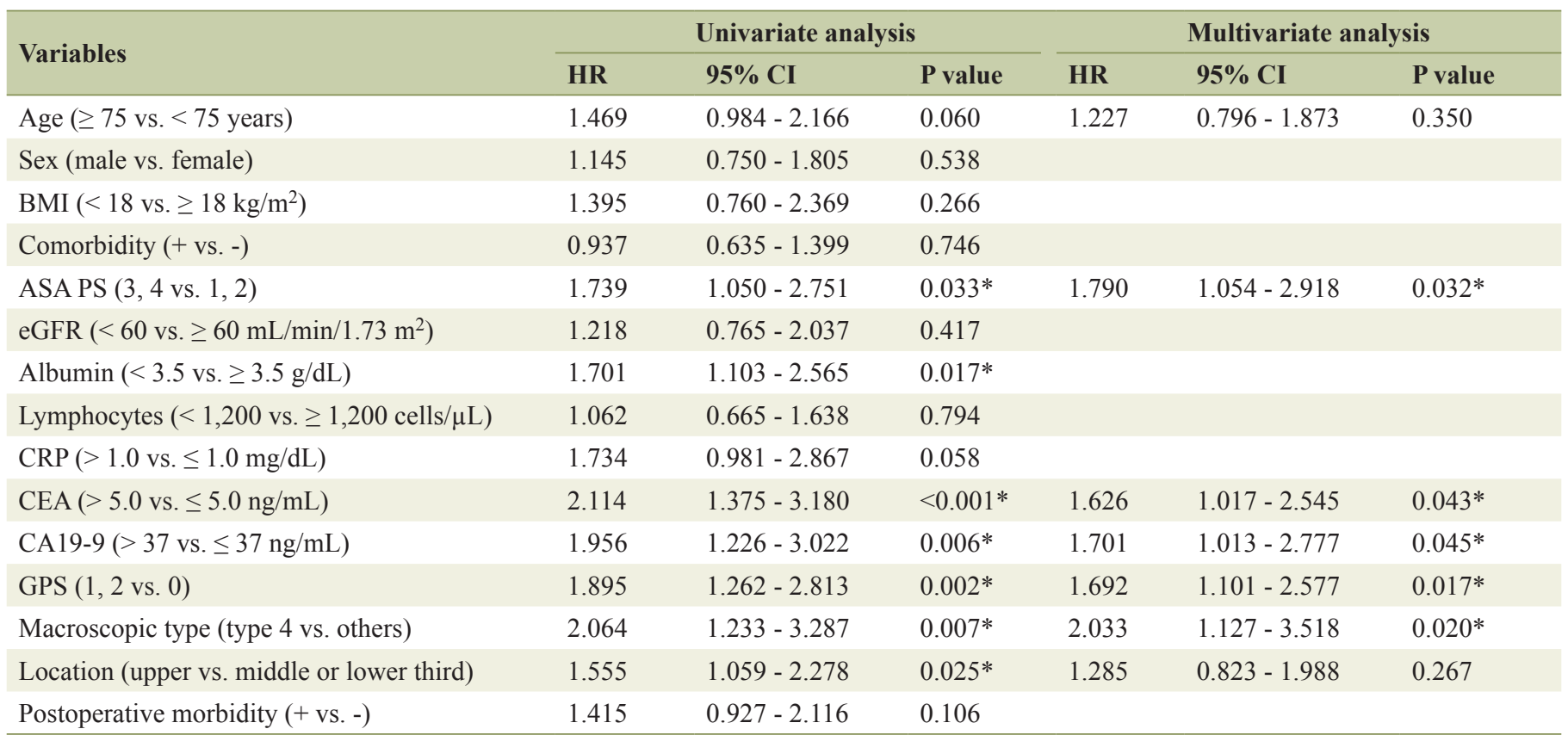

HR: hazard ratio; Cl: confidence interval; BMI: body mass index; ASA PS: American Society of Anesthesiologists physical status; eGFR: estimated glomerular filtration rate; CRP: C-reactive protein; CEA: carcinoembryonic antigen; CA19-9: carbohydrate antigen 19-9; GPS: Glasgow prognostic score.

nally developed as a new prognostic indicator for patients with inoperable stage III and IV non-small-cell lung cancer $[8,9]$. This combination of factors has rendered the GPS a more sensitive predictive marker of survival than either CRP or albumin alone. In addition to lung cancer, reports have described GPS as a useful prognostic marker for various types of cancer, including colorectal [11, 23-25] and pancreatic cancers [26]. Additionally, studies have increasingly shown an association between the GPS and survival in patients with gastric cancer

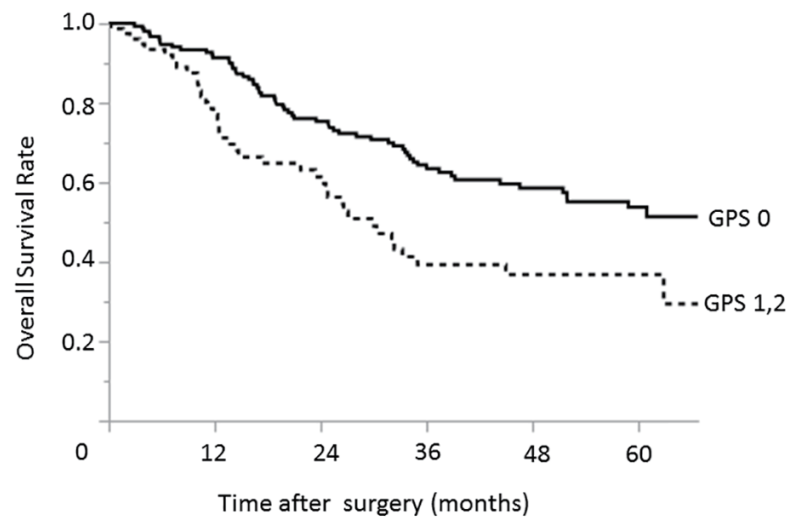

Figure 2. Relationship between the GPS and overall survival among patients with advanced-stage (pStage II, III, and IV) gastric cancers. The 3- and 5-year overall survival rates among patients with GPS of 0 were $63.5 \%$ and $53.8 \%$, respectively; the corresponding rates among patients with GPSs of $1-2$ were $39.3 \%$ and $36.8 \%$, respectively. GPS: Glasgow prognostic score.
[10-20].

The GPS can be calculated easily from preoperative laboratory data and exhibits prognostic sensitivity equivalent to that of the tumor markers CEA and CA19-9. Although the pathological stage is very important to prognostic stratifications of cancer patients, decisions regarding the indications of postoperative adjuvant therapy, or follow-up planning, this information can be achieved postoperatively. In contrast, the GPS may facilitate preoperative treatment decision-making, including surgical procedure planning.

Previous reports have described the association of systemic inflammatory responses with poor prognosis, as well as several possible underlying mechanisms; however, this link remains incompletely understood. Elevated levels of serum CRP or higher GPSs have been reported to correlate with elevated levels of serum cytokines, including interleukins 6 and 8, and vascular endothelial growth factor-A, which promote angiogenesis in cancer lesions $[27,28]$. Furthermore, cancer patients with acute-phase responses, indicated by elevated serum CRP levels, exhibit compromised drug metabolism consequent to reduced cytochrome P450 3A function [29]. This enzyme is responsible for the metabolism of various chemotherapy agents, and dysregulation might cause poor tolerability [9] and subsequent poor responses [30] to chemotherapy.

Although the GPS has largely been reported as a prognostic factor for patients with very advanced cancers, several authors suggested that this score could be applied to patients with early-stage gastric cancer. Nozoe et al [12] reported the applicability of the GPS even for gastric cancer patients with early-stage tumors (stage I: 132/232 patients), but did not an- 
alyze the prognostic factors relevant to patients with stage I disease separately from those with advanced-stage disease. In a retrospective analysis of 1,710 patients with operable gastric cancer, Jiang et al [10] reported that the modified GPS is an easily available prognostic indicator. These authors also reported a significant survival difference among stage I gastric cancer patients, depending on the modified GPS. However, Jiang and colleagues used only a univariate analysis comprising a Kaplan-Meier analysis and log-rank test to demonstrate this survival difference, and did not conduct a multivariate analysis. Furthermore, their study included only six patients with stage I disease and a modified GPS of 2 . In our study, we not only conducted a multivariate analysis of all gastric cancer patients, but also stratified patients by early-stage (stage I) and advanced-stage disease (stage II, III, and IV) for separate analyses and found that GPS was not a significant prognostic factor in the early-stage group.

In our study, the clinicopathological characteristics differed considerably between of patients in the early-stage and advanced-stage groups. In particular, we observed significant intergroup differences in potential prognosticators such as age, serum albumin and serum CRP levels, tumor marker expression, tumor location, and GPS. The early-stage group included significantly younger patients and had lower frequencies of hypoalbuminemia, elevated serum CRP and tumor marker levels, high GPSs, tumors located in the upper third of the stomach, and type 4 gastric cancers, as well as a lower postoperative morbidity rate. This suggests that it would be inappropriate to analyze patients with gastric cancer as a whole. Notably, the prognostic factors also differed considerably between the two groups. In the univariate analysis, many factors, including the ASA PS, serum albumin level, tumor marker levels, GPS, and tumor location, were identified as significant prognosticators in advanced-stage group, whereas, only the CA19-9 level was significant in the early-stage group. We attribute this discrepancy to the generally localized state of early-stage gastric cancers, and the ability to cure most such cases by surgery alone, even in the presence of unfavorable factors. In our series, the 3- and 5-year overall survival rates among 295 patients with stage I gastric cancer who were treated with surgery alone (after excluding nine patients who received adjuvant chemotherapy) were $96.1 \%$ and $94.2 \%$, respectively.

The limitations of this study included the retrospective, single-institute design, and the inclusion of gastric cancer patients who underwent gastrectomy with either curative or palliative intent. In other words, patients were determined to have resectable disease, at least with palliative intent, and to be able to withstand surgery. Accordingly, we excluded patients who were deemed inoperable because of a very locally advanced disease, a poor general status caused by a very advanced cancer, or severe comorbidities. This selection bias might have led to a low proportion of patients with a GPS of $2(20 / 544$ patients $(3.7 \%))$. Similar to our study, Jiang et al [10] also studied patients with operable gastric cancers and reported a frequency of GPS 2 cases of 3.9\% (67/1,710). In contrast, Crumley et al [13] reported a frequency of $17.4 \%(45 / 258)$ in a study that included inoperable gastroesophageal cancers, and Forrest et al [8] reported a frequency of 20.5\% (33/161 patients) in a cohort that included inoperable non-small-cell lung cancer patients. Second, the median follow-up duration was 38.6 months, which was insufficiently long to assess verylong term outcome (e.g., 5-year follow-up results). However, the differences of prognostic factors between stage I and advanced-stage gastric cancers were evident even during this abbreviated follow-up period.

In conclusion, GPS is a simple, useful prognostic score that can be calculated at the time of diagnosis from routine laboratory data, and may facilitate treatment decision-making for patients with gastric cancer, especially those with advancedstage disease. However, it does not appear to be applicable to patients with stage I gastric cancer.

\section{Conflict of Interest}

The authors declare that they have no conflict of interest.

\section{References}

1. Jackson KV, Ginsburg ES, Hornstein MD, Rein MS, Clarke RN. Multinucleation in normally fertilized embryos is associated with an accelerated ovulation induction response and lower implantation and pregnancy rates in in vitro fertilization-embryo transfer cycles. Fertil Steril. 1998;70(1):60-66.

2. Nashimoto A, Akazawa K, Isobe Y, Miyashiro I, Katai H, Kodera Y, Tsujitani S, et al. Gastric cancer treated in 2002 in Japan: 2009 annual report of the JGCA nationwide registry. Gastric Cancer. 2013;16(1):1-27.

3. Kurita N, Miyata H, Gotoh M, Shimada M, Imura S, Kimura W, Tomita N, et al. Risk model for distal gastrectomy when treating gastric cancer on the basis of data from 33,917 Japanese patients collected using a nationwide web-based data entry system. Ann Surg. 2015;262(2):295-303.

4. Watanabe M, Miyata H, Gotoh M, Baba H, Kimura W, Tomita N, Nakagoe T, et al. Total gastrectomy risk model: data from 20,011 Japanese patients in a nationwide internet-based database. Ann Surg. 2014;260(6):1034-1039.

5. Onate-Ocana LF, Aiello-Crocifoglio V, Gallardo-Rincon D, Herrera-Goepfert R, Brom-Valladares R, Carrillo JF, Cervera E, et al. Serum albumin as a significant prognostic factor for patients with gastric carcinoma. Ann Surg Oncol. 2007;14(2):381-389.

6. Lien YC, Hsieh CC, Wu YC, Hsu HS, Hsu WH, Wang LS, Huang MH, et al. Preoperative serum albumin level is a prognostic indicator for adenocarcinoma of the gastric cardia. J Gastrointest Surg. 2004;8(8):1041-1048.

7. Crumley AB, McMillan DC, McKernan M, Going JJ, Shearer CJ, Stuart RC. An elevated C-reactive protein concentration, prior to surgery, predicts poor cancer-specific survival in patients undergoing resection for gastrooesophageal cancer. Br J Cancer. 2006;94(11):1568-1571.

8. Forrest LM, McMillan DC, McArdle CS, Angerson WJ, Dunlop DJ. Evaluation of cumulative prognostic scores based on the systemic inflammatory response in patients with inoperable non-small-cell lung cancer. Br J Cancer. 
2003;89(6):1028-1030.

9. Forrest LM, McMillan DC, McArdle CS, Angerson WJ, Dunlop DJ. Comparison of an inflammation-based prognostic score (GPS) with performance status (ECOG) in patients receiving platinum-based chemotherapy for inoperable non-small-cell lung cancer. Br J Cancer. 2004;90(9):1704-1706.

10. Jiang X, Hiki N, Nunobe S, Kumagai K, Kubota T, Aikou S, Sano T, et al. Prognostic importance of the inflammation-based Glasgow prognostic score in patients with gastric cancer. Br J Cancer. 2012;107(2):275-279.

11. Proctor MJ, Morrison DS, Talwar D, Balmer SM, O’Reilly DS, Foulis AK, Horgan PG, et al. An inflammation-based prognostic score (mGPS) predicts cancer survival independent of tumour site: a Glasgow Inflammation Outcome Study. Br J Cancer. 2011;104(4):726-734.

12. Nozoe T, Iguchi T, Egashira A, Adachi E, Matsukuma A, Ezaki T. Significance of modified Glasgow prognostic score as a useful indicator for prognosis of patients with gastric carcinoma. Am J Surg. 2011;201(2):186-191.

13. Crumley AB, McMillan DC, McKernan M, McDonald AC, Stuart RC. Evaluation of an inflammation-based prognostic score in patients with inoperable gastro-oesophageal cancer. Br J Cancer. 2006;94(5):637-641.

14. Melling N, Gruning A, Tachezy M, Nentwich M, Reeh M, Uzunoglu FG, Vashist YK, et al. Glasgow Prognostic Score may be a prognostic index for overall and perioperative survival in gastric cancer without perioperative treatment. Surgery. 2016;159(6):1548-1556.

15. Aurello P, Tierno SM, Berardi G, Tomassini F, Magistri P, D'Angelo F, Ramacciato G. Value of preoperative inflammation-based prognostic scores in predicting overall survival and disease-free survival in patients with gastric cancer. Ann Surg Oncol. 2014;21(6):1998-2004.

16. Dutta S, Crumley AB, Fullarton GM, Horgan PG, McMillan DC. Comparison of the prognostic value of tumour and patient related factors in patients undergoing potentially curative resection of gastric cancer. Am J Surg. 2012;204(3):294-299.

17. Hirashima K, Watanabe M, Shigaki H, Imamura Y, Ida S, Iwatsuki M, Ishimoto T, et al. Prognostic significance of the modified Glasgow prognostic score in elderly patients with gastric cancer. J Gastroenterol. 2014;49(6):10401046.

18. Wang DS, Ren C, Qiu MZ, Luo HY, Wang ZQ, Zhang DS, Wang FH, et al. Comparison of the prognostic value of various preoperative inflammation-based factors in patients with stage III gastric cancer. Tumour Biol. 2012;33(3):749-756.

19. Elahi MM, McMillan DC, McArdle CS, Angerson WJ,
Sattar N. Score based on hypoalbuminemia and elevated C-reactive protein predicts survival in patients with advanced gastrointestinal cancer. Nutr Cancer. 2004;48(2):171-173.

20. Jeong JH, Lim SM, Yun JY, Rhee GW, Lim JY, Cho JY, Kim YR. Comparison of two inflammation-based prognostic scores in patients with unresectable advanced gastric cancer. Oncology. 2012;83(5):292-299.

21. Japanese Gastric Cancer A. Japanese gastric cancer treatment guidelines 2014 (ver. 4). Gastric Cancer. 2017;20(1):1-19.

22. Japanese Gastric Cancer A. Japanese classification of gastric carcinoma: 3rd English edition. Gastric Cancer. 2011;14(2):101-112.

23. Leitch EF, Chakrabarti M, Crozier JE, McKee RF, Anderson JH, Horgan PG, McMillan DC. Comparison of the prognostic value of selected markers of the systemic inflammatory response in patients with colorectal cancer. Br J Cancer. 2007;97(9):1266-1270.

24. Ishizuka $M$, Nagata $H$, Takagi $K$, Kubota $K$. Influence of inflammation-based prognostic score on mortality of patients undergoing chemotherapy for far advanced or recurrent unresectable colorectal cancer. Ann Surg. 2009;250(2):268-272.

25. Roxburgh CS, Salmond JM, Horgan PG, Oien KA, McMillan DC. Comparison of the prognostic value of inflammation-based pathologic and biochemical criteria in patients undergoing potentially curative resection for colorectal cancer. Ann Surg. 2009;249(5):788-793.

26. Shimoda M, Katoh M, Kita J, Sawada T, Kubota K. The Glasgow Prognostic Score is a good predictor of treatment outcome in patients with unresectable pancreatic cancer. Chemotherapy. 2010;56(6):501-506.

27. Krzystek-Korpacka M, Matusiewicz M, Diakowska D, Grabowski K, Blachut K, Kustrzeba-Wojcicka I, Terlecki $\mathrm{G}$, et al. Acute-phase response proteins are related to cachexia and accelerated angiogenesis in gastroesophageal cancers. Clin Chem Lab Med. 2008;46(3):359-364.

28. Kim DK, Oh SY, Kwon HC, Lee S, Kwon KA, Kim BG, $\mathrm{Kim} \mathrm{SG}$, et al. Clinical significances of preoperative serum interleukin-6 and C-reactive protein level in operable gastric cancer. BMC Cancer. 2009;9:155.

29. Rivory LP, Slaviero KA, Clarke SJ. Hepatic cytochrome P450 3A drug metabolism is reduced in cancer patients who have an acute-phase response. $\mathrm{Br} \mathrm{J}$ Cancer. 2002;87(3):277-280.

30. Shimada H, Kitabayashi H, Nabeya Y, Okazumi S, Matsubara H, Funami Y, Miyazawa Y, et al. Treatment response and prognosis of patients after recurrence of esophageal cancer. Surgery. 2003;133(1):24-31. 